\title{
Spatial Distribution of Cold Antihydrogen Formation
}

N. Madsen, ${ }^{1}$ M. Amoretti, ${ }^{2}$ C. Amsler, ${ }^{3}$ G. Bonomi, ${ }^{4}$ P. D. Bowe,${ }^{5}$ C. Carraro, ${ }^{2}$ C. L. Cesar, ${ }^{6}$ M. Charlton, ${ }^{5}$ M. Doser, ${ }^{4}$ A. Fontana, ${ }^{7,8}$ M. C. Fujiwara,,${ }^{9,10}$ R. Funakoshi, ${ }^{9}$ P. Genova, ${ }^{7,8}$ J. S. Hangst, ${ }^{1}$ R. S. Hayano, ${ }^{9}$ L. V. Jørgensen, ${ }^{5}$ A. Kellerbauer, ${ }^{4}$ V. Lagomarsino, ${ }^{2,11}$ R. Landua, ${ }^{4}$ E. Lodi-Rizzini, ${ }^{7,12}$ M. Macri, ${ }^{2}$ D. Mitchard, ${ }^{5}$ P. Montagna, ${ }^{7,8}$ H. Pruys, ${ }^{3}$ C. Regenfus, ${ }^{3}$ A. Rotondi, ${ }^{7,8}$ G. Testera, ${ }^{2}$ A. Variola, ${ }^{2}$ L. Venturelli, ${ }^{12}$ D. P. van der Werf, ${ }^{5}$ Y. Yamazaki, ${ }^{10}$ and N. Zurlo ${ }^{12}$

(ATHENA Collaboration)

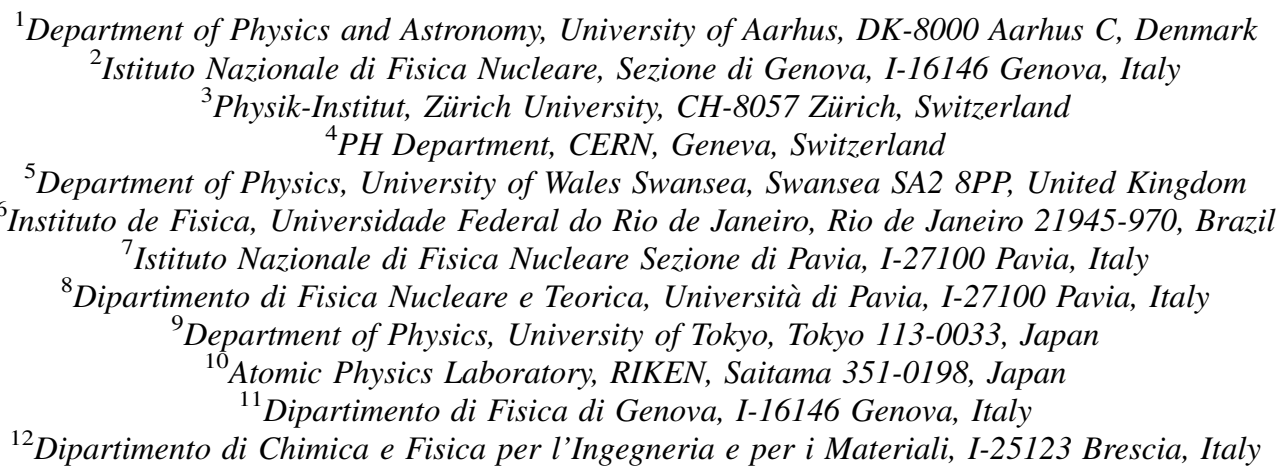
(Received 5 October 2004; published 27 January 2005)

\begin{abstract}
Antihydrogen is formed when antiprotons are mixed with cold positrons in a nested Penning trap. We present experimental evidence, obtained using our antihydrogen annihilation detector, that the spatial distribution of the emerging antihydrogen atoms is independent of the positron temperature and axially enhanced. This indicates that antihydrogen is formed before the antiprotons are in thermal equilibrium with the positron plasma. This result has important implications for the trapping and spectroscopy of antihydrogen.
\end{abstract}

Cold antihydrogen $(\overline{\mathrm{H}})$ formation was reported by the ATHENA Collaboration [1] at the CERN Antiproton Decelerator [2] in September 2002. Similar results were reported shortly thereafter by the ATRAP Collaboration [3]. Both experiments form the $\overline{\mathrm{H}}$ atoms by mixing samples of antiprotons $(\bar{p})$ with positrons $\left(e^{+}\right)$in a nested Penning trap.

The spatial distribution of the emerging $\overline{\mathrm{H}}$ atoms can give crucial insights into the nature and dynamics of the formation process, and by inference the distribution of states formed. More knowledge of the latter, and how to influence it, is needed in order to prepare states that can be trapped and studied. For instance, precision spectroscopy of $\overline{\mathrm{H}}$ building on accurate hydrogen spectroscopy $[4,5]$ promises high precision $C P T$ tests. In this Letter we present evidence, using the directly measured spatial distribution, that $\overline{\mathrm{H}}$ is formed before the $\bar{p}$ attain thermal equilibrium with the $e^{+}$.

The ATHENA mixing trap, comprising a series of hollow cylinders, has the axial $(z)$ potential configuration of a nested Penning trap, which permits two plasmas of opposite charge to come into contact [6]. A 3 T axial magnetic field ensures transverse confinement of the particles. The mixing trap is kept at $\sim 15 \mathrm{~K}$. A detector for $\bar{p}$ and $e^{+}$annihilations surrounds the trap in barrel geometry.
Details of the experimental apparatus can be found in Ref. [7].

Antihydrogen production is carried out by first loading the mixing trap with $\sim 7 \times 10^{7} e^{+}$, which cool to the cryogenic temperature by the emission of synchrotron radiation, and then by injecting about $10^{4} \bar{p}$ that interact through the Coulomb interaction with the $e^{+}$plasma. When $\bar{p}$ are mixed with cryogenic $e^{+}$we refer to the procedure as "cold" mixing. Alternatively, the $e^{+}$plasma may be heated by radio frequency excitation [8]. Mixing with a plasma where the temperature $\left(T_{e^{+}}\right)$has been increased by $3500 \mathrm{~K}$ we term "hot" mixing.

We let the $\bar{p}$ interact with the $e^{+}$for about $180 \mathrm{~s}$ before ejecting both species and restarting the cycle. A $\bar{p}$ may undergo one of several fates. Apart from forming $\overline{\mathrm{H}}$, a $\bar{p}$ may annihilate on residual gas, drift to and annihilate on the cylindrical walls, or survive until the final dump of all species. Neutral $\overline{\mathrm{H}}$ atoms drift away from the formation region until they annihilate on the electrodes of the mixing trap. The $\overline{\mathrm{H}}$ detector can observe charged particle tracks, and from these we can reconstruct the vertices of $\bar{p}$ annihilations. The detector also observes the $511 \mathrm{keV}$ photons from the $e^{+}$annihilation [1,9].

In earlier work we relied on the simultaneous annihilation of both $\bar{p}$ and a $e^{+}$in order to detect $\overline{\mathrm{H}}$ production [1]. 
Detection of $e^{+}$annihilations is inefficient and limits $\overline{\mathrm{H}}$ detection efficiency to less than $0.25 \%$, whereas $\bar{p}$ annihilations have about $25 \%$ chance of being detected and reconstructed. Using only $\bar{p}$ vertices may thus significantly enhance our detection efficiency. Recently we showed that the bulk of the $\bar{p}$ annihilation events we observe during cold mixing of $\bar{p}$ and $e^{+}$stem from $\overline{\mathrm{H}}$ annihilations [10]. This was demonstrated by comparing the results from cold mixing with those from hot mixing, where $\overline{\mathrm{H}}$ production is suppressed [1]. The difference in the number of $\bar{p}$ annihilations between cold and hot mixing was shown to be due entirely to $\overline{\mathrm{H}}$ production.

Using the detector we can extract the spatial distribution of the observed $\overline{\mathrm{H}}$ annihilations. Earlier we showed that the $\overline{\mathrm{H}}$ distribution projected into a plane perpendicular to the trap axis $(z)$ is rotationally symmetric [1]. No time dependent change of the $\overline{\mathrm{H}}$ distribution during individual mixing cycles has been observed. The time resolution of changes in the $\overline{\mathrm{H}}$ spatial distribution is limited by statistics to $\sim 1 \mathrm{~s}$. Figure 1(a) shows the $z$ distribution of $\bar{p}$ annihilation vertices from 305 full cold mixing cycles. The distribution

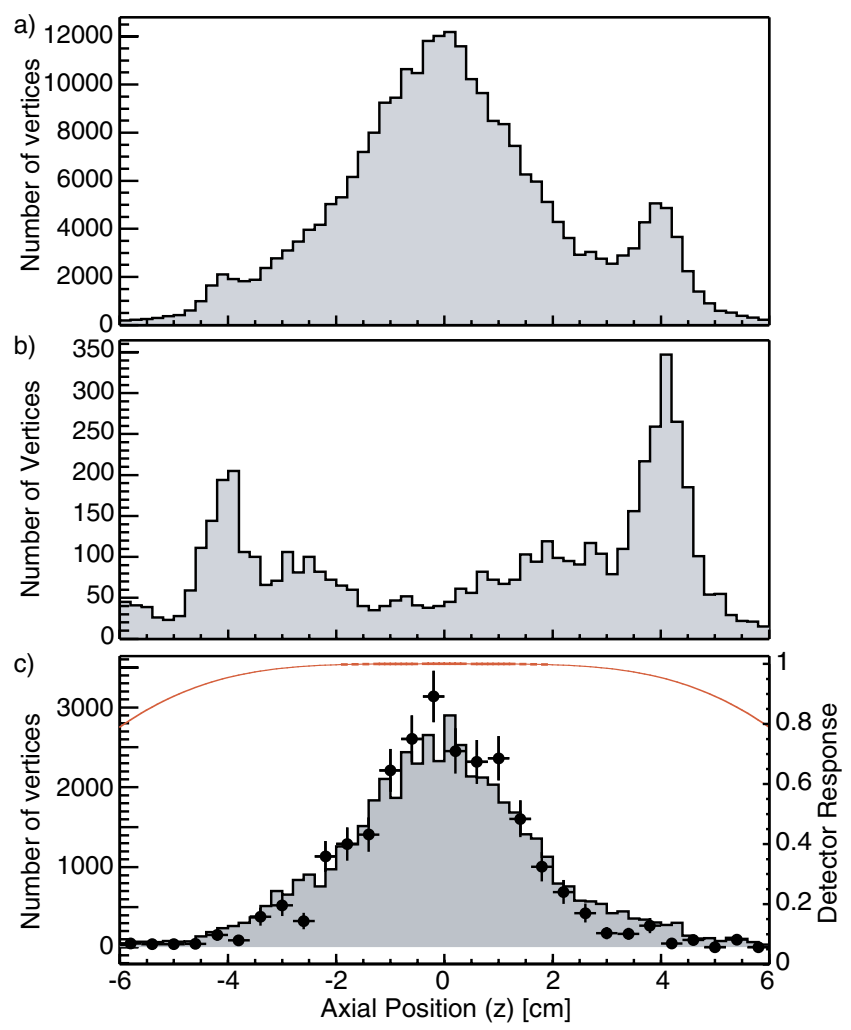

FIG. 1 (color online). Axial $(z)$ vertex distributions for (a) all vertices during cold mixing $\left(1.75 \times 10^{5}\right.$ vertices). (b) Axial vertex distributions for $\bar{p}$ annihilating in a nested trap without $e^{+}$(5717 vertices). (c) Shaded area: Cold mixing with hot mixing subtracted and using a spatial cut of $x>0$ and $y>0$ (see text) $\left(4.9 \times 10^{4}\right.$ vertices). Full circles: $\bar{p}$ annihilations with two back-to-back $511 \mathrm{keV}$ photons during cold mixing (488 events, scaled to the area under the shaded curve). Solid line: Detector response function from Monte Carlo simulations. has two distinct features. The first is a broad, smooth, peaked distribution. The second consists of the two smaller peaks on either side of the distribution. We have determined that the latter peaks are due to $\bar{p}$-only annihilations because they disappear when selecting events with two back-to-back $511 \mathrm{keV}$ photons and are also present in the hot mixing distribution where $\overline{\mathrm{H}}$ production is negligible.

For comparison we also recorded the distribution of annihilations when the $\bar{p}$ were injected into a nested trap without $e^{+}$, so that there was no $\overline{\mathrm{H}}$ production. Figure 1(b) shows the $z$ distribution of annihilations from 24 mixing cycles under these circumstances. We observe the small peaks seen on the side of the cold mixing distribution and no broad central feature.

The $\bar{p}$-only annihilations in the two peaks are also localized in the transverse plane. This phenomenon, which makes it possible to spatially separate $\overline{\mathrm{H}}$ annihilations from $\bar{p}$-only annihilations, is discussed in detail in Ref. [11]. In the region $x>0$ and $y>0$, where $x$ and $y$ are the horizontal and vertical directions, respectively, we observe no $\bar{p}$-only annihilations in the measurements presented here. To increase the signal to background ratio on $\overline{\mathrm{H}}$ data we remove $\bar{p}$-only annihilations by selecting only vertices from this region. The resulting $z$ distribution with hot mixing subtracted is shown in Fig. 1(c). The hot mixing was normalized to the same beam intensity as the cold. For comparison we also show the $z$ distribution of $\bar{p}$ annihilations that were accompanied by simultaneous back-to-back $511 \mathrm{keV}$ gammas from $e^{+}$annihilation using the same selection criteria as in Ref. [1]. The good agreement between these two distributions means that we can use the full cold mixing distribution with the $x y$ cut applied and the hot mixing subtracted (the shaded distribution in Fig. 1(c)] as the distribution of $\overline{\mathrm{H}}$ annihilations. The detector response function, shown in Fig. 1(c), is the efficiency of the detector for $\bar{p}$ annihilation events on the trap wall (electrodes). The response function was calculated by Monte Carlo by evenly distributing $\bar{p}$ annihilations on the trap walls and simulating the detector response [7].

Using the same method as above we have also extracted the axial distributions for $\overline{\mathrm{H}}$ produced with heated samples of $e^{+}$. Figure 2 shows the axial distribution for $\overline{\mathrm{H}}$ annihilations on the walls for cold mixing [from Fig. 1(c)] and for measurements with the $e^{+}$plasma heated by 175 and $500 \mathrm{~K}$. There is no change in the distribution as a function of $e^{+}$temperature, although the total number of events varies strongly [12]. Also shown in Fig. 2 is a simple calculation of the $z$ distribution resulting from isotropic emission of $\overline{\mathrm{H}}$ from the ellipsoidal positron plasma.

The $e^{+}$plasma is typically $2.5 \mathrm{~mm}$ in radius $\left(r_{e^{+}}\right)$, is $32 \mathrm{~mm}$ in length $\left(l_{e^{+}}\right)$, and has a density of $n_{e^{+}}=1.7 \times$ $10^{8} \mathrm{~cm}^{-3}$ [8]. The thermal velocities of $15 \mathrm{~K} e^{+}$and $\bar{p}$ are $v_{\mathrm{th}}^{e^{+}} \sim 1.5 \times 10^{4} \mathrm{~m} \mathrm{~s}^{-1}$ and $v_{\mathrm{th}}^{\bar{p}} \sim 350 \mathrm{~m} \mathrm{~s}^{-1}$, respectively.

The space charge of the $e^{+}$plasma generates a radial $\mathbf{E}$ field and causes the equilibrium state of the cold plasma to 


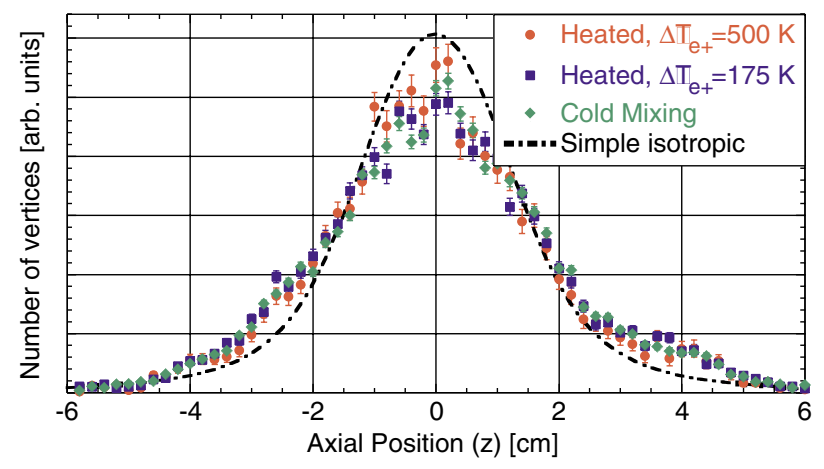

FIG. 2 (color). Axial $\overline{\mathrm{H}}$ distributions for cold mixing and mixing with $e^{+}$heated by two different amounts (hot mixing subtracted, $x y$ cut applied). The dot-dashed line is a simple calculation of isotropic emission from the $e^{+}$plasma volume. The distributions have been normalized to the same area.

be one of laminar rotational flow. With our typical plasma parameters, the frequency of rotation is $\sim 80 \mathrm{kHz}$, corresponding to a surface velocity of $1.3 \times 10^{3} \mathrm{~m} \mathrm{~s}^{-1}$. Temperatures are thus given in this rotating frame. A $\bar{p}$ in the trap exhibits cyclotron rotation around the magnetic field lines with a frequency of $46 \mathrm{MHz}$. A radial electric field causes a guiding-center $\mathbf{E} \times \mathbf{B}$ drift motion in the azimuthal direction, independent of charge and mass. However, the difference in mass between $\bar{p}$ and $e^{+}$causes a difference in centrifugal potential such that the $\bar{p}$ tends to separate outwards. For our densities and temperatures this effect is small and slow (separation time $\sim 1000 \mathrm{~s}[13,14]$ ) compared to the $\overline{\mathrm{H}}$ production rate. We thus consider the antiproton radial distribution to be static during antihydrogen production. The $\bar{p}$ move axially in and out of the $e^{+}$ plasma as time passes. The axial oscillation frequency at injection is $\sim 100 \mathrm{kHz}$, small compared to the cyclotron frequency which sets the time scale for the onset of the $\mathbf{E} \times \mathbf{B}$ drift motion. We thus simplify the analysis by assuming that $\bar{p}$ inside the $e^{+}$plasma, where $\overline{\mathrm{H}}$ may be formed, rotate with the positrons. In the following, thermal equilibrium refers to a state where $T_{\bar{p}}=T_{e^{+}}$in this rotating frame.

The $\bar{p}$ were precooled by being embedded in an electron $\left(e^{-}\right)$plasma of similar dimensions as the $e^{+}$plasma. The $e^{-}$are ejected before the $\bar{p}$ are mixed with the $e^{+}$. Detailed studies of the cooling of the $\bar{p}$ by the $e^{+}$in our apparatus suggest that the $\bar{p}$ cloud is radially larger than the $e^{+}$ plasma [15]. As the rotation velocity in the $e^{+}$plasma is small compared to $v_{\mathrm{th}}^{e^{+}}$and $v_{\mathrm{th}}^{e^{+}} \gg v_{\mathrm{th}}^{\bar{p}}$, it has negligible influence on the $\overline{\mathrm{H}}$ formation rate. We therefore assume that $\overline{\mathrm{H}}$ is formed homogeneously throughout the rotating ellipsoidal $e^{+}$plasma. Another scenario would be formation on the surface of the $e^{+}$plasma only, something one could imagine if the centrifugal separation was fast. This only strengthens the arguments to follow. With these reflections in mind we henceforth assume homogeneous formation throughout the $e^{+}$plasma.
We can model the axial distribution by randomly distributing $\overline{\mathrm{H}}$ in a selected formation volume and assigning to each $\overline{\mathrm{H}}$ a velocity from a three-dimensional Gaussian velocity distribution characterized by transverse $\left(T_{\bar{p}}^{\perp}\right)$ and axial $\left(T_{\bar{p}}^{\|}\right)$temperatures and an azimuthal velocity given by the radial position of the $\overline{\mathrm{H}}$. We use two different temperatures to describe nonequilibrium conditions. The intersection of the $\overline{\mathrm{H}}$ undisturbed path with the cylindrical electrodes is then calculated. Then the vertex reconstruction resolution of the detector $(\sigma=4 \mathrm{~mm})$ and the response function are folded onto the result. The $\bar{p}$, due to their mass, dominate the momentum of the $\overline{\mathrm{H}}$, and we therefore neglect $T_{e^{+}}$in this model.

Figure 3 shows a number of calculated distributions using the model described above. Also shown is the measured cold mixing distribution. The model does not reproduce the observations with $T_{\bar{p}}^{\|}=T_{\bar{p}}^{\perp}$. A longer $e^{+}$plasma gives a wider distribution, but the necessary length $(\sim 60 \mathrm{~mm})$ to match the observed distribution is much larger than that measured. If we assume $T_{\bar{p}}^{\perp}=15 \mathrm{~K}$, the model matches the observed cold mixing distribution with $T_{\bar{p}}^{\|}=(10 \pm 2) \times T_{\bar{p}}^{\perp}$ (solid curve in Fig. 3). This gives a lower limit of $T_{\bar{p}}^{\|}=150 \mathrm{~K}$. The $\bar{p}$ that form $\overline{\mathrm{H}}$ are therefore not in thermal equilibrium with the $e^{+}$. We cannot determine $T_{\bar{p}}$ of the $\bar{p}$ that form $\overline{\mathrm{H}}$ from these measurements. However, as we increase $T_{\bar{p}}^{\perp}$ the necessary difference between the $T_{\bar{p}}^{\|}$and $T_{\bar{p}}^{\perp}$ to model the observations decreases asymptotically to a factor $2.3 \pm 0.6$ (shown in Fig. 3). This is because the influence of $\mathbf{E} \times \mathbf{B}$ rotation on the distribution decreases with increasing temperature. Thus even with no influence from $\mathbf{E} \times \mathbf{B}$ rotation we cannot find consistency with thermal equilibrium; i.e., our conclusion is independent of the absolute $e^{+}$temperature. Comparing the model distributions we find that for

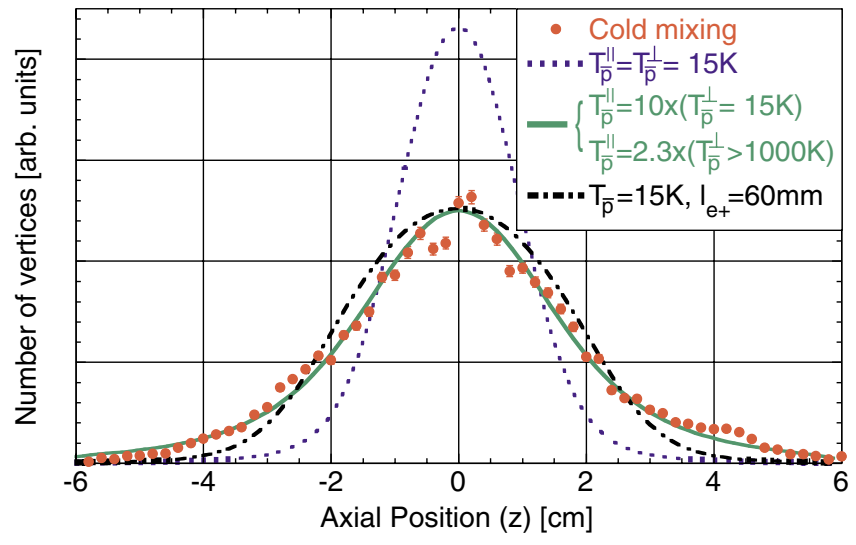

FIG. 3 (color). Comparison of the axial distribution from cold mixing with a number of calculated distributions. Standard $e^{+}$ plasma parameters and $\mathbf{E} \times \mathbf{B}$ rotation were used except for the dot-dashed curve where $l_{e^{+}}=60 \mathrm{~mm}$. Homogeneous formation in the plasma was assumed. 
$T_{\bar{p}} \geq 10^{3} \mathrm{~K}$ the influence of the $\mathbf{E} \times \mathbf{B}$ rotation is negligible. If $T_{e^{+}}<10^{3} \mathrm{~K}$, and the $\bar{p}$ are in thermal equilibrium with the $e^{+}$, the $\overline{\mathrm{H}}$ distribution should thus change when the $e^{+}$are heated. We observe no change (Fig. 2) also indicating that the $\bar{p}$ forming $\overline{\mathrm{H}}$ are not in thermal equilibrium with the $e^{+}$.

That the $\overline{\mathrm{H}}$ is formed before thermal equilibrium between $\bar{p}$ and $e^{+}$is achieved indicates that the formation rate of $\overline{\mathrm{H}}$ is high compared to the $\bar{p}$ cooling rate. The formation rate is expected to scale inversely with some power of the relative velocity [12]. In the literature this rate is often given as a function of the temperature, as thermal equilibrium between the particle species is assumed. Because of the much larger mass of the $\bar{p}$, the relative velocity is dominated by the $e^{+}$velocity as long as $T_{\bar{p}} \lesssim$ $2000 \times T_{e^{+}}$. For $T_{e^{+}}=15 \mathrm{~K}$ this is equivalent to $T_{\bar{p}}=$ $30000 \mathrm{~K}$ or a $\bar{p}$ kinetic energy of $2.6 \mathrm{eV}$. The $\bar{p}$ injection energy is $\sim 15 \mathrm{eV}$. Our earlier studies of the dynamics of $\bar{p}$ cooling during $\overline{\mathrm{H}}$ formation were not precise enough to resolve whether $\overline{\mathrm{H}}$ formation sets in at a relative energy of less than $2.6 \mathrm{eV}$, as they resolved only the energy spread of the $\bar{p}$ to the $\sim 2 \mathrm{eV}$ level. [15]. In those experiments the cooling time was measured to be $10-20 \mathrm{~ms}$, in good agreement with recent theoretical estimates [16]. For comparison, an estimate of the 3-body formation rate using Ref. [17], using $10^{4} \bar{p}$ and our $e^{+}$plasma parameters gives a rate of $\sim 8 \mathrm{kHz}$ at a temperature of $30 \mathrm{~K}$, roughly equivalent to the relative velocities of $30000 \mathrm{~K} \bar{p}$ interacting with $15 \mathrm{~K}^{+}$. This rate is very high compared to the cooling rate and allows many $\bar{p}$ to form $\overline{\mathrm{H}}$ at high temperature. Recent simulations by Robicheaux indicate that $\overline{\mathrm{H}}$ is formed with high axial momentum (i.e., high $T_{\bar{p}}^{\|}$) [18].

The high production temperature found could explain the high $\overline{\mathrm{H}}$ velocities measured by the ATRAP Collaboration [19]. They measured velocities equal to an $\overline{\mathrm{H}}$ temperature of $\sim 2 \times 10^{3} \mathrm{~K}$. Their method, however, measures only weakly bound $\overline{\mathrm{H}}$ atoms that can be field ionized by a static electric field $\left(\sim 75 \mathrm{~V} \mathrm{~cm}^{-1}\right)$. Furthermore, the $\bar{p}$ were actively driven through a $e^{+}$ plasma with many fewer $e^{+}$and much lower density than in this experiment. In the experiments presented here almost all $\overline{\mathrm{H}}$ that may survive the combined electric fields of the trap and the $e^{+}$plasma are observed [12].

If, indeed, the $\overline{\mathrm{H}}$ are formed before equilibrium as our results indicate, it could be an obstacle to the trapping of $\overline{\mathrm{H}}$, and thus precision $\overline{\mathrm{H}}$ spectroscopy. Typical neutral traps have depths about $1 \mathrm{~K}$. If $\overline{\mathrm{H}}$ atoms are formed at some $10^{2} \mathrm{~K}$, very few will be trapped. Lowering $T_{e^{+}}$may not help as formation is fast compared to the cooling. To produce cold $\overline{\mathrm{H}}$, it is thus necessary to have cold $\bar{p}$ before mixing with $e^{+}$. A possible solution could be to invert the current mechanism for formation. That is, trap the $\bar{p}$ in the center of the nested trap, possibly with some electrons to keep them cold, and then pass $e^{+}$through them, reinjecting them regularly as has already been done with $\bar{p}$ [20]. Positronium formation or axial separation of $\bar{p}$ and $e^{+}$ are both potential obstacles in this scheme. An alternative scheme that should also produce $\overline{\mathrm{H}}$ at or close to ambient temperature would be to form $\overline{\mathrm{H}}$ by $\bar{p}$ positronium collisions [21,22].

To summarize, we have studied the spatial distribution of the $\overline{\mathrm{H}}$ annihilations during mixing of $\bar{p}$ and $e^{+}$. We find that the distribution of $\overline{\mathrm{H}}$ emerging from the formation region is independent of the $e^{+}$temperature and enhanced in the axial direction. The latter assumes homogeneous formation throughout the $e^{+}$plasma, and rotation of the $\bar{p}$ with the $e^{+}$. We argue that this indicates that $\overline{\mathrm{H}}$ is not formed under conditions of thermal equilibrium between the $e^{+}$and the $\bar{p}$. The lower limit of the formation temperature is $T_{\bar{p}}^{\|}=150 \mathrm{~K}$ and $T_{\bar{p}}^{\perp}=15 \mathrm{~K}$. This result indicates that the nested trap scheme as currently deployed is inadequate for producing $\overline{\mathrm{H}}$ that may be magnetically trapped and used for precision spectroscopy. More studies are needed to determine to what extent this problem may be circumvented.

This work was supported by CNPq, FAPERJ, CCMN/ UFRJ (Brazil), INFN (Italy), MEXT (Japan), SNF (Switzerland), SNF (Denmark), and EPSRC (U.K.).

[1] M. Amoretti et al., Nature (London) 419, 456 (2002).

[2] S. Maury, Hyperfine Interact. 109, 43 (1997).

[3] G. Gabrielse et al., Phys. Rev. Lett. 89, 213401 (2002).

[4] M. Niering et al., Phys. Rev. Lett. 84, 5496 (2000).

[5] C. L. Cesar et al., Phys. Rev. Lett. 77, 255 (1996).

[6] G. Gabrielse et al., Phys. Lett. A 129, 38 (1988).

[7] M. Amoretti et al., Nucl. Instrum. Methods Phys. Res., Sect. A 518, 679 (2004).

[8] M. Amoretti et al., Phys. Rev. Lett. 91, 055001 (2003).

[9] C. Regenfus, Nucl. Instrum. Methods Phys. Res., Sect. A 501, 65 (2003).

[10] M. Amoretti et al., Phys. Lett. B 578, 23 (2004).

[11] M.C. Fujiwara et al., Phys. Rev. Lett. 92, 065005 (2004).

[12] M. Amoretti et al., Phys. Lett. B 583, 59 (2004).

[13] T. M. O’Neil, Phys. Fluids 24, 1447 (1981).

[14] T. M. O'Neil (private communication).

[15] M. Amoretti et al., Phys. Lett. B 590, 133 (2004).

[16] Y. Chang and C. A. Ordonez, Phys. Rev. E 69, 037401 (2004).

[17] M.E. Glinsky and T. M. O’Neil, Phys. Fluids B 3, 1279 (1991).

[18] F. Robicheaux, Phys. Rev. A 70, 022510 (2004).

[19] G. Gabrielse et al., Phys. Rev. Lett. 93, 073401 (2004).

[20] G. Gabrielse et al., Phys. Rev. Lett. 89, 233401 (2002).

[21] J. W. Humberston et al., J. Phys. B 20, L25 (1987).

[22] M. Charlton, Phys. Lett. A 143, 143 (1990). 